\title{
Visual Alphabets on Different Levels of Abstraction for the Recognition of Deformable Objects
}

\author{
Martin Stommel $^{1}$ and Klaus-Dieter Kuhnert ${ }^{2}$ \\ 1 TZI Center for Computing and Communication Technologies, \\ University Bremen, Am Fallturm 1, 28359 Bremen, Germany \\ mstommel@tzi.de \\ 2 Institute of Real-Time Learning Systems, University of Siegen \\ Hoelderlinstrasse 3, 57076 Siegen, Germany \\ kuhnert@fb12.uni-siegen.de
}

\begin{abstract}
Recognition systems for complex and deformable objects must handle a variety of possible object appearances. In this paper, a compositional approach to this problem is studied which splits the set of possible appearances into easier sub-problems. To this end, a grammar is introduced that represents objects by a hierarchy of increasingly abstract visual alphabets. These alphabets store features, complex patterns and different views of objects. The geometrical constraints are optimised to the respective level of abstraction. The performance of the method is demonstrated on a cartoon data base with high intra-class variance.
\end{abstract}

\section{Introduction}

Many recent studies are based on compositional approaches where objects are modeled as parts in comparably loose geometric relationships [1341220]. The idea is to tolerate geometric distortions to a certain degree but to model the characteristic features of an object still correctly. The geometrical constraints are formulated statistically 204] or structural [1. Most research on compositional object models covers the training of single object views (e.g. 4]). While this is appropriate for objects that occur preferentially in a certain distinctive pose, it fails for deformable or moving objects. The trade-off between a bag-offeatures model and precise geometric constellations [314] in the image plane is often discussed. Hierarchical models that cover geometric constellations on different levels of abstraction on the other hand are rare, so possible important dependencies remain widely unidentified. To account for noise or the high intraclass variances of deformable objects, the training methods are usually based on flexible matching procedures. Probabilistic approaches are preferred to exact graph matching procedures 7/5/22].

Although the modeling of the local and global geometry has also been addressed by researchers with a stronger computer graphics background (e.g. [16]), the parts of the compositional models are mostly represented by local descriptors in the shape of feature vectors 1011176 . Heuristical learning or clustering 
methods (e.g. 981211]) are used to condense the feature set, possibly tuning it to a particular application [21. The resulting feature sets constitute a certain parallel to the alphabet of moderately complex features found by Tanaka [19] in the inferior temporal (IT) cortex. However, the question how these cells work together to build an internal multi-view object representation, has not been answered yet. Nielsen et al. [15] indicate that monkeys represent rotated objects by different sets of features for different angles of the rotation. Humans however seem to use another mechanism. Miyashita et al. 1314 report that cells in the IT of a monkey could be trained to sequences of arbitrary patterns and conclude that this mechanism could be used to learn different appearances of a single object.

The work presented here takes these considerations into account. In order to recognise deformable objects in multiple views, we propose a hierarchical model that represents objects by visual alphabets on different levels of abstraction. This allows for a more accurate trade-off between geometry and the part characteristics.

\section{Noise-Tolerant Syntactical Model}

Inspired by Han and Zhu's [5] recognition procedure, we use an attribute grammar for object modelling. Significant differences consist in the visual primitives, the production rules and the noise handling, though. Let

$$
\mathcal{G}=\left(A_{N}, A_{T}, R, S\right)
$$

denote our attribute grammar consisting of a visual alphabet $A_{N}$ of non-terminal elements, a visual alphabet $A_{T}$ of terminal elements including the empty word $\varepsilon$, a set of rules $R$ and a root element $S \in A_{N}$.

The terminal elements are the visual primitives that describe our objects. We use edge points, corners and skeleton points as visual primitives, since there are many suitable feature detectors with known performance. Edge points are further parameterised by their orientation. Skeleton points are parameterised by the orientation of the local skeleton line, the local image intensity and the distance to the nearest edge. The different parameterisations are enumerated and each parameterisation is assigned a single terminal element.

Terminal elements are grouped to complex parts of objects which are represented by the non-terminal elements. Non-terminal elements can in turn be grouped to more complex parts or whole objects.

Terminal and non-terminal elements are attributed by a vector

$$
g=(x, y, \theta, \sigma, w)
$$

which stores the image position $x, y$ of a recognised visual element as well as trained (attributes $\theta$ and $\sigma$ ) and temporary $(w)$ information about the recognition process. 
Non-terminal elements are expanded to strings of terminal and non-terminal elements using production rules of the form

$$
a_{0} \rightarrow a_{1} a_{2} a_{3} \ldots a_{n} \mid \epsilon, \quad a_{0} \in A_{N}, a_{1}, a_{2}, a_{3} \ldots a_{n} \in A_{T} \cup A_{N},
$$

defining a context free grammar, basically. Each rule is however associated with a number of constraint equations of the general form

$$
f_{i}\left(x\left(a_{0}\right)\right)=g_{i}\left(x\left(a_{1}\right), x\left(a_{2}\right), x\left(a_{3}\right), \ldots\right), \quad i=1,2,3, \ldots
$$

where $f_{i}$ and $g_{i}$ are projection functions on the attributes of the terminal and non-terminal elements [5]. These functions specify the geometrical relationship between visual elements and guide an acyclic, bottom up recognition procedure. Top down information passing could support rule expansions on lower levels of abstraction by providing information about the surrounding. This is an option for future work, however, and the recognition already works without it. Figure 1 illustrates the usage of the production rules.

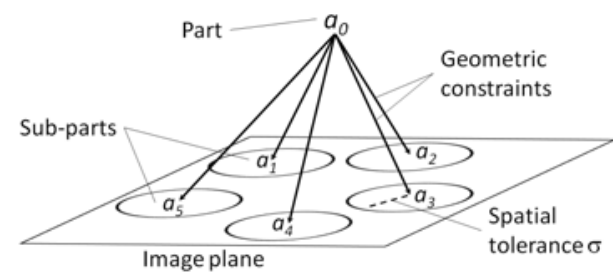

Fig. 1. Illustration of a production rule $a_{0} \rightarrow a_{1} a_{2} a_{3} a_{4} a_{5}$. The sub-parts are arranged in certain relative positions, which are modeled by geometric constraints. To account for deformations, small displacements within a tolerance $\sigma$ are allowed.

The design of the grammar takes into account that due to noise, elements on the right side of a production rule may not be found in an image or that they are spuriously assigned to a wrong element. Noise in the parameterisation of the base features is handled by additional production rules. If an element $a_{2}$ is likely to be mistaken for an element $a_{3}$, the alternative production rules

$$
a_{0} \rightarrow a_{1} a_{2} \mid a_{1} a_{3}
$$

are created.

If a non-terminal or terminal from the right side of a production rule is not detected in an image, the non-terminal on the left side cannot be expanded. To relax this situation by admitting such missing elements, new rules could be introduced that enumerate all valid subsets of elements on the right side of the original rule. This however would enlarge the rule set unnecessarily.

Instead, special constraint equations are introduced that compensate for missing elements. The aim of these equations is to transfer information about the 
presence or absence of a certain visual element into the attribute $w$ of a nonterminal element. For the expansion to single terminal elements, this yields the production rules and constraint equations

$$
\begin{aligned}
& a_{0} \rightarrow a_{1}, w\left(a_{0}\right)=1 \\
& a_{0} \rightarrow \varepsilon, w\left(a_{0}\right)=0, \quad a_{0} \in A_{N}, a_{1} \in A_{T} .
\end{aligned}
$$

The non-terminal $a_{0}$ only expands into a terminal if its weight $w$ is equal to 1 . Otherwise it indicates a missing feature.

To handle missing non-terminal elements, the geometry must be taken into account. In our approach, geometric constraints define a relative position $d_{x}, d_{y}$ between two elements $a_{0}, a_{i} \in A_{N}$, which could be expressed by a constraint equation like

$$
\operatorname{dist}\left(x\left(a_{0}\right), y\left(a_{0}\right), x\left(a_{i}\right)+d_{x}, y\left(a_{i}\right)+d_{y}\right)<\sigma\left(a_{0}\right),
$$

where $a_{0}$ is on the left side of a production rule, $a_{i}$ is on the right side, and dist is a suitable distance measure (e.g. the $L_{\max }$-distance). Noise tolerance is introduced by demanding that the number of correctly detected elements on the right side of the production rule exceeds the threshold $\theta$ in the attribute vector of the element on the left side of the rule. This results in the production rules and constraint equations

$$
\begin{aligned}
a_{0} & \rightarrow a_{1} a_{2} a_{3} \ldots, \\
\theta\left(a_{0}\right) & \leq \sum_{i \geq 1} \begin{cases}w\left(a_{i}\right) & \text { if eq. } 8 \text {. } a_{1}, \cdots \in A_{N} \\
0 & \text { otherwise }\end{cases} \\
w\left(a_{0}\right) & =1
\end{aligned}
$$

for the correct matching of a part $a_{0}$ to a number of sub-parts $a_{1}, a_{2}, a_{3}, \ldots$, and

$$
a_{0} \rightarrow \epsilon, w\left(a_{0}\right)=0
$$

for non-terminal $a_{0}$ that is not recognised. Equation 10 asserts that the expansion 9 is only valid if a sufficient number of non-terminals on the right side of the production rule is detected. Both the presence of a sub-part (indicated by $w\left(a_{i}\right)=1$ ) as well as the geometry are checked. Otherwise, only the expansion into the empty word $\epsilon$ is possible. The non-terminal on the left side is then characterised by the attribute $w=0$ (eq.112).

Object recognition works by evaluating the constraint equations bottom up from the level of features, over the level of parts, over the level of object poses and appearances, to the level of object classes until the root element $S$ of the grammar is reached. For the root element, a constraint equation demands the attribute value $w(S)=1$. All visual elements that yield a weight of $w=1$ are regarded as recognised. Elements with a weight $w=0$ mark the (near) end of a rule expansion. They occur only if a part of an object can not be matched. 


\section{Training on Different Levels of Abstraction}

The training of the model addresses the visual alphabets $A_{N}$ and $A_{T}$, the noise suppressing parameters $\sigma$ and $\theta$ of the attribute vectors, and the geometrical constraints $d_{x}$ and $d_{y}$ of the production rules. The importance of geometrical constraints compared to the feature type is judged differently in the literature. While Weber, Perona et al. 20 promote a highly restrictive constellation model, Crandall et al. 2] and Fergus [4 report a better performance for models with small clique-sizes, i.e. fewer part dependencies. Stommel and Kuhnert [18 find out that the geometrical constraints also depend on the level of abstraction. Therefore we decide to use a differenciated training procedure, that is tuned to the depth of the rule expansion. The training is carried out on the cartoon data base described later. Figure 2 gives an overview.

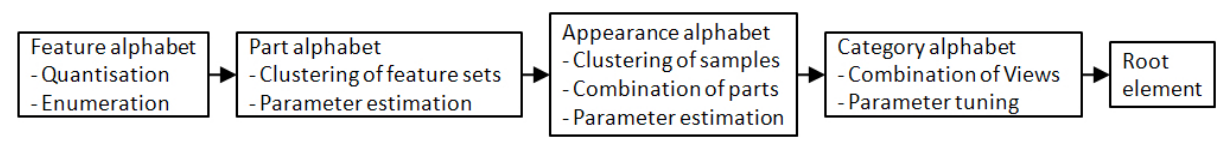

Fig. 2. The Training process comprises the definition of 4 visual alphabets on different levels of abstraction. Taken together they form $A_{N} \cup A_{T}$. The production rules assert that non-terminals can only be expanded to less abstract elements.

\subsection{Terminal Elements}

The deepest level of expansion concerns the terminal elements. They represent edge pieces in different orientations, corners, and center points of homogeneous areas (skeletonisation) with differing intensity, diameter and orientation. The quantisation intervals for the feature parameters are optimised with respect to the information contents measured in a trial classification task. This yields a terminal alphabet of about 1200 elements and the corresponding noise suppressing non-terminals (eq. [6]and 7). The dual integration of edges and the skeletonisation widens the area of application.

\subsection{Non-terminals for Object Parts}

The non-terminals from the previous step are now grouped to complex object parts. For every part, a non-terminal and the corresponding production rule is created. Since statistical measurements on feature co-occurrences by Stommel and Kuhnert 18 show that spatial proximity is crucial for the frequency of a feature combination in a sample, the creation of more complex visual alphabets is based on the principle of locality.

First, groups of spatially proximate feature vectors are identified in the sample images. To obtain a compact part alphabet with high information capacity, these candidate patterns are clustered with regard to their mutual similarity. To this end, every pattern is described by a non-terminal and the corresponding rules that relate it to its terminal features. The relative feature positions are expressed 


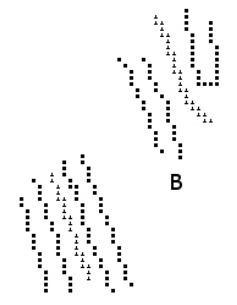

A
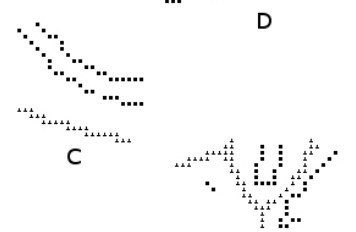

$\mathrm{E}$

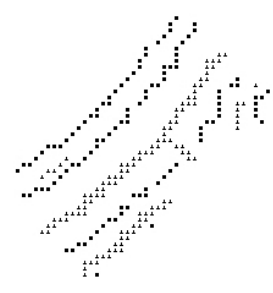

$\mathrm{F}$

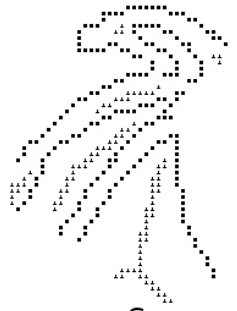

G

Fig. 3. Patterns from the part alphabet (corresponding to right rule sides): Two patterns from one cluster (patterns A, B). Cluster prototypes of size 20 (C, D, E) and 40 pixels (F, G). Square markings represent edge features, triangles area features.

by constraint equations. Two pattern are regarded similar if the non-terminal of one pattern can be expanded to the terminal elements of the other one. For every resulting cluster, one pattern that is compatible to all other patterns in the cluster is included in the part alphabet. The resulting set consists of about 5000 non-terminals for patterns with a diameter of 10 to 60 pixels.

Initial clusterings showed a high dependency between the threshold $\theta$ and the spatial tolerance $\sigma$ of the attribute vector. Since high thresholds provided the most exact localisations, a fixed threshold $\theta=90 \%$ is chosen for all parts. Further optimisations are done via the remaining free parameter $\sigma$, for which an good linear approximation based on the diameter of a pattern is found. Figure 3 shows elements from the resulting part alphabet.

\subsection{Non-terminals for Object Appearances}

Next, a visual alphabet is trained that abstracts from object pose and appearance. Distinct appearances are found by hierarchically clustering the training images into homogeneous groups. Every group is modeled by a non-terminal together with a production rule that maps it to suitable elements of the part alphabet. The resulting new non-terminals thus constitute a visual alphabet that enumerates the poses and appearances of single objects. The approach is partly neurophysiologically motivated 131415$]$.

Since the right rule sides consist of elements from the part alphabet, the appearance clustering is based on the number of parts that occur jointly in two samples. The occurrence is measured in terms of possible rule expansions.

The result of the clustering is a dendrogram that represents possible groupings of sample images with respect to intersecting part occurrences. Figure 4 illustrates the procedure. For 800 training images and about 5000 patterns in the part alphabet, the dendrogram has a depth of 15 .

Next, a set of nodes from the dendrogram must be chosen for modeling and the non-terminals and production rules must be parameterised with respect to their attributes and geometrical constraints. 
The production rules are constructed by sub-sampling the image positions where parts can be found for all images of a group. For every such position and part, the corresponding non-terminal is added to the right side of the production rule. The geometric constraint is defined accordingly.

The nodes of the dendrogram that are selected for modeling should at least cover all training samples to maximise recall. Overlapping groupings introduce redundancy that increases the robustness to noise at the cost of a bigger model.

Experiments with single appearance models identify the position (depth and height, fig. 4) of a node in the dendrogram as a crucial variable for the parameterisation of the model and the resulting model size (tab.11): Appearance models for nodes near the root of the dendrogram quickly grow infeasibly large or do not achieve a high precision during classification. It is however possible to restrict the parameter space to a region where a linear model for the noise suppression attribute $\sigma$ is applicable and the model size stays within treatable limits. The threshold $\theta$ is optimised with respect to a minimum number of false matches. Outliers in the histogram of the precision of appearance models are removed, since they increase the false positive rate.

\subsection{Non-terminal Elements for Categorisation}

A last category alphabet is introduced that builds a layer between the root element $S$ and the appearance alphabet. The purpose of this alphabet is to use the redundancy within the appearance alphabet for the optimisation of the recognition system to different configurations in terms of precision and recall. In the

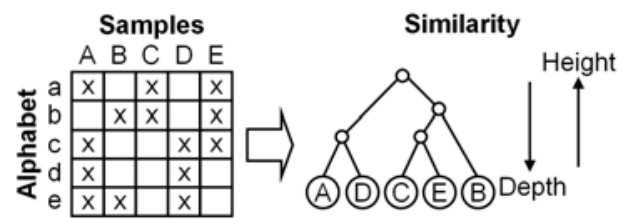

Fig. 4. Clustering of object appearances. The table shows which elements of the part alphabet can be expanded to features of a certain sample image by the application of production rules. Samples with similar parts are grouped together. Related groups are represented as nodes in a dendrogram. The height and depth of the nodes are crucial for the parameterisation of the model.

Table 1. Correlation between model parameters and the position of nodes in the appearance dendrogram (fig. (4). The correlation coefficient is normalised to $\left[\begin{array}{ll}-1 & 1\end{array}\right]$.

\begin{tabular}{llr} 
1st Variable & 2nd Variable & Correlation \\
\hline Std. deviation size & Size & 0.79 \\
Optimal threshold $\theta$ Height & 0.73 \\
Optimal threshold $\theta$ Depth & -0.70 \\
Spatial tolerance $\sigma$ & Height & 0.92 \\
Spatial tolerance $\sigma$ & Depth & -0.69 \\
Number of parts & Spatial tolerance $\sigma$ & 0.81
\end{tabular}


following, we present two example configurations to achieve either a high suppression of false matches or a high number of detections. Since the appearance models already represent whole objects, the geometrical constraints are set to $d_{x}=d_{y}=0$, i.e. all production rules on this level function as pixelwise bags of features. The elements of the category alphabet represent different classes of objects and therefore the end of the classification process. A sample is assigned the predominant class of all category nodes that have the attribute $w=1$ after the recognition process.

The first model configuration aims at the suppression of false matches. To this end, for every foreground sample a non-terminal category element is defined that combines all elements of the appearance alphabet that are consistent with this sample. A certain proportion of the resulting category alphabet will also respond to samples from wrong classes. To suppress false matches, the thresholds $\theta$ of the category non-terminals are set to an upper bound of the noise level which is estimated practically as the maximum number of non-terminals on the right side of a rule that simultaneously recognise wrong samples.

The second model configuration aims at a higher rate of recognised positive samples. To this end, the threshold $\theta$ of every non-terminal from the category alphabet is optimised towards the maximum accuracy measured over all samples. The resulting lower thresholds increase the likelihood of a category element to be expanded to a sample image. The category alphabet is finally pruned until the maximum accuracy is achieved.

\section{Data Base and Test Results}

The cartoon data base consists of 1600 images showing the head of a cartoon character and 1600 background images of equal size. The background images do not show any cartoon heads. Figure[5 gives an impression of the diversity

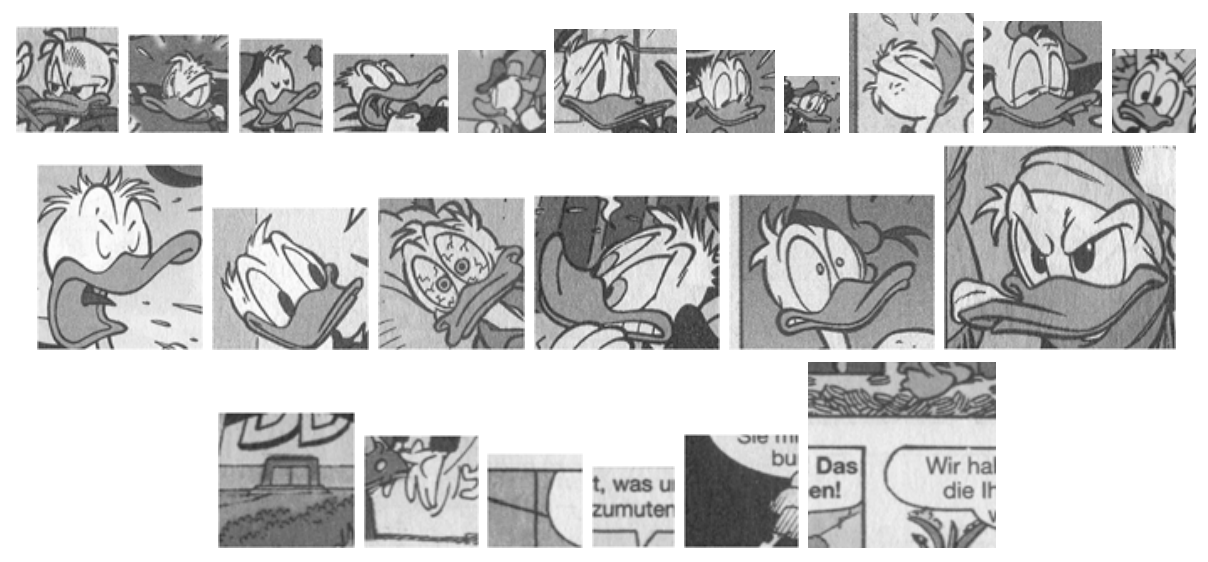

Fig. 5. Samples from the cartoon data base. The upper rows show foreground samples, the bottom row background samples. 
of object poses and geometric deformations. The seemingly clear boundaries between adjacent areas are often affected by misalignments between different printing colours. Intensity variations occur across one cartoon page, but also within small areas due to half-tone printing, and between different pages. The data base is thus sufficiently difficult to validate the recognition system. Foreground and background images are randomised and split into equally sized training and test sets. For the first model configuration a precision of $97 \%$, a recall of $57 \%$ and an accuracy of $78 \%$ is achieved. The recognition is thus very reliable, but also rather selective. The second model configuration yields a recall of $89 \%$, a precision of $72 \%$ and an overall accuracy of $77 \%$. The general performance of the model is thus equally high but the recall is significantly higher and the classifier works less conservative.

\section{Conclusion}

An object recognition system is presented that combines a syntactical model and statistical training method. The structure of the underlying grammar is noise resistent and based on both the geometric properties of feature co-occurrence statistics, as well as on neurophysiological findings about pose representation. Since the training method creates visual alphabets on different levels of abstraction, crucial dependencies on the depth of the rule expansion are taken into account. A data base with images of a strongly deformable cartoon character is used to test the method. Two exemplary model configurations are presented which tune the model either towards a high precision of $97 \%$ or a high recall of $89 \%$. A rate of correct classifications of $77 \%-78 \%$ for both configurations demonstrates the performance of the method.

\section{References}

1. Behmo, R., Paragios, N., Prinet, V.: Graph Commute Times for Image Representation. In: IEEE Conference in Computer Vision and Pattern Recognition, CVPR 2008 (2008)

2. Crandall, D.J., Felzenszwalb, P.F., Huttenlocher, D.P.: Spatial Priors for PartBased Recognition Using Statistical Models. In: IEEE Conference on Computer Vision and Pattern Recognition (CVPR), pp. 10-17 (2005)

3. Crandall, D.J., Huttenlocher, D.P.: Composite Models of Objects and Scenes for Category Recognition. In: IEEE Conference on Computer Vision and Pattern Recognition, CVPR (2007)

4. Fergus, R., Perona, P., Zisserman, A.: A Sparse Object Category Model for Efficient Learning and Complete Recognition. In: Ponce, J., Hebert, M., Schmid, C., Zisserman, A. (eds.) Toward Category-Level Object Recognition. LNCS, vol. 4170, pp. 443-461. Springer, Heidelberg (2006)

5. Han, F., Zhu, S.C.: Bottom-up/Top-Down Image Parsing by Attribute Graph Grammar. In: Proceedings of the Tenth IEEE International Conference on Computer Vision (ICCV), vol. 2, pp. 1778-1785 (2005) 
6. Ke, Y., Sukthankar, R.: PCA-SIFT: A more distinctive representation for local image descriptors. Technical Report IRP-TR-03-15, School of Computer Science, Carnegie Mellon University (2004)

7. Lee, W.-J., Duin, R.: An Inexact Graph Comparison Approach in Joint Eigenspace. In: da Vitoria Lobo, N., Kasparis, T., Roli, F., Kwok, J.T., Georgiopoulos, M., Anagnostopoulos, G.C., Loog, M. (eds.) S+SSPR 2008. LNCS, vol. 5342, pp. 35-44. Springer, Heidelberg (2008)

8. Lin, Z., Hua, G., Davis, L.: Multiple Instance Feature for Robust Part-based Object Detection. In: IEEE Conference on Computer Vision and Pattern Recognition, CVPR (2009)

9. Liu, J., Yang, Y., Shah, M.: Learning Semantic Visual Vocabularies Using Diffusion Distance. In: IEEE Conference on Computer Vision and Pattern Recognition, CVPR (2009)

10. Lowe, D.G.: Object Recognition from Local Scale-Invariant Features. In: Proc. of the International Conference on Computer Vision (ICCV), Kerkyra, Greece, September 1999, vol. 2, pp. 1150-1157 (1999)

11. Mikolajczyk, K., Leibe, B., Schiele, B.: Local Features for Object Class Recognition. In: International Conference on Computer Vision ICCV 2005 (October 2005)

12. Mikolajczyk, K., Leibe, B., Schiele, B.: Multiple Object Class Detection with a Generative Model. In: IEEE Conference on Computer Vision and Pattern Recognition (CVPR), June 2006, vol. 1, pp. 26-36 (2006)

13. Miyashita, Y., Sakai, K., Higuchi, S.-I., Masui, N.: Localization of Primal LongTerm Memory in the Primate Temporal Cortex. In: Squire, L.R., Weinberger, N.M., Lynch, G., McGaugh, J.L. (eds.) Memory: Organization And Locus of Change (1991)

14. Miyashita, Y.: Inferior Temporal Cortex: Where Visual Perception Meets Memory. Annual Reviews of Neuroscience 16, 245-265 (1993)

15. Nielsen, K.J., Logothetis, N.K., Rainer, G.: Object features used by humans and monkeys to identify rotated shapes. Journal of Vision 8(2), 1-15 (2008)

16. Salzmann, M., Urtasun, R., Fua, P.: Local Deformation Models for Monocular 3D Shape Recovery. In: IEEE Conference on Computer Vision and Pattern Recognition, CVPR (2008)

17. Stark, M., Schiele, B.: How good are local features for classes of geometric objects. In: IEEE 11th International Conference on Computer Vision ICCV, pp. 1-8 (2007)

18. Stommel, M., Kuhnert, K.-D.: A Hierarchical Model for the Recognition of Deformable Objects. In: Int'l Conf. on Computer Vision and Graphics 2008 (ICCVG 2008), Warsaw, Poland, November 10-12 (2008)

19. Tanaka, K.: Inferotemporal cortex and object vision. Annual Reviews of Neuroscience 19, 109-139 (1996)

20. Weber, M., Welling, M., Perona, P.: Unsupervised Learning of Models for Recognition. In: Vernon, D. (ed.) ECCV 2000. LNCS, vol. 1842, pp. 628-641. Springer, Heidelberg (2000)

21. Yang, L., Jin, R., Sukthankar, R., Jurie, F.: Unifying Discriminative Visual Codebook Generation with Classifier Training for Object Category Recognition. In: IEEE Conference on Computer Vision and Pattern Recognition, CVPR (2008)

22. Zass, R., Shashua, A.: Probabilistic Graph and Hypergraph Matching. In: IEEE Conference on Computer Vision and Pattern Recognition, CVPR (2008) 